\title{
Protein Nutrition Index as a Function of Patient Survival Rate in Peritoneal Dialysis
}

\author{
Kuan-Hsing Chen ${ }^{a, b} \quad$ Ching-Herng Wu ${ }^{c} \quad$ Ching-Wei Hsu ${ }^{a, b} \quad$ Yu-Ming Chen ${ }^{a, b}$ \\ Shu-Man Weng ${ }^{a}$ Chih-Wei Yang ${ }^{a, b}$ Cheng-Chieh Hung ${ }^{a, b}$ \\ aDivision of Nephrology, Chang Gung Memorial Hospital, and ${ }^{\mathrm{b}}$ School of Medicine, Chang Gung University, \\ Taoyuan, and 'Division of Nephrology, Poh-Ai Hospital, Yi-Lan, Taiwan, ROC
}

\section{Key Words}

Protein energy wasting $\cdot$ Albumin $\cdot$ Normalized protein nitrogen appearance $\cdot$ Percent lean body mass $\cdot$ Mortality $\cdot$ Peritoneal dialysis

\begin{abstract}
Background/Aims: Protein-energy wasting (PEW) is a wellknown risk factor of long-term survival in peritoneal dialysis (PD) patients. Serum albumin is a measure of visceral protein, lean body mass is a measure of somatic protein stores and normalized protein nitrogen appearance is a measure of daily protein intake. A protein nutrition index (PNI) that combined these 3 factors was designed and tested as a function of survival in PD patients. Methods: We enrolled 552 PD patients for this study. Demographic, biochemical, nutritional markers, comorbidity and dialysis-related data were obtained. The PNI was calculated. All patients were followed up to investigate the risks for mortality. Results: Patients with probable PEW/low-average nutrition were older and had lower serum creatinine $(\mathrm{Cr})$ and blood urea nitrogen, lower adequacy data and higher D4/P4 Cr compared with patients with high-average/good nutrition. 108 patients (19.6\%) died during the observational period. By multivariate analysis, we found only age, comorbidity index and PNI (relative risk = 0.84 , confidence interval: $0.76-0.93, p=0.001$ ) to be independent predictors of mortality. Conclusion: The PNI at the start of PD is associated with all-cause mortality, and each
\end{abstract}

increase by a score of 1 in PNI leads to a $16 \%$ decrease in the risk of mortality. Predialysis evaluation of this scoring system is recommended for further research in order to improve outcomes in PD patients.

Copyright $\odot 2010$ S. Karger AG, Basel

\section{Introduction}

Protein-energy wasting (PEW) is defined as reduced somatic and/or circulating body protein mass, decreased fat mass and (usually) reduced protein and energy intake, according to the expert panel of the International Society of Renal Nutrition and Metabolism [1]. Protein-energy malnutrition is reserved to cases with low nutritional intake. PEW has been associated with increased morbidity and mortality in patients with dialysis. Previously, the CANUSA study revealed that decreased serum albumin and poor nutritional status increased the risk of death [2]. PEW has been associated with increased morbidity and mortality in patients with chronic kidney disease [3, 4]. Multiple factors, such as disturbances in protein-energy metabolism, infections, chronic inflammation, hormonal derangements, comorbidities and poor intake caused by uremic toxins may be responsible for PEW [1].

Several studies have provided indirect evidence of a link between survival of peritoneal dialysis (PD) patients and nutritional status at the start of PD. Besides the CA- 
NUSA study, Hakim and Lazarus [5] suggested that malnutrition at the initiation of dialysis is a strong predictor of subsequent increase in the relative risk (RR) of death. de Mutsert et al. [6] also reported that PEW at baseline assessed with subjective global assessment (SGA) was associated with a twofold increased mortality risk in 7 years of follow-up in chronic dialysis patients.

The diagnosis of PEW requires several clinical, nutritional and biochemical measures in individuals with kidney disease. However, the criteria are complicated. An alternative nutritional scoring system may help to diagnose PEW. Although SGA reflects overall the nutritional status, measures such as lean body mass (LBM) and normalized protein nitrogen appearance (nPNA) would be more representative of the somatic protein store and daily protein intake [7]. Serum albumin is a measure of visceral protein and is the nutritional marker most often used. Protein malnutrition may decrease nPNA within days to weeks, serum albumin within weeks to months, and LBM within months to years.

These three factors may actually present different statuses of protein nutrition. In a previous study, Gurreebun et al. [8] reported that measurement of SGA did not lead to the diagnosis of malnutrition in patients in whom this had not already been potentially identified by the measurement of serum albumin and body mass index (BMI). Therefore, we believed that measures of nPNA, percent LBM (\%LBM), and serum albumin may represent the true protein nutritional status of patients. A protein nutrition index (PNI) was thus designed. In this study, we proved that PNI at the start of dialysis can predict longterm survival in $\mathrm{PD}$ patients.

\section{Patients and Methods}

This clinical study followed the Declaration of Helsinki and was approved by the Medical Ethics Committee of the Chang Gung Memorial Hospital, Taipei.

From January 1994 to December 2004, a total of 552 incident uremic patients, who were over 18 years of age and maintained on PD for more than 3 months at Chang Gung Memorial Hospital were included in this retrospective study. At $57 \pm 21$ days after the initiation of $\mathrm{PD}$, the patients underwent evaluations of serum blood urea nitrogen (BUN), creatinine (Cr), albumin, nPNA, \%LBM, comorbid diseases, adequacy of PD and a peritoneal equilibration test. Clinical outcomes were determined by mortality.

Serum BUN and Cr were determined by standard techniques. Serum albumin was determined by the bromocresol green method.

Dietary protein intake was estimated from the protein equivalent of total nitrogen appearance (PNA) using the Randerson
Table 1. Protein nutrition index system

\begin{tabular}{llccl}
\hline & Score 4 & Score 3 & Score 2 & Score 1 \\
\hline Albumin, g/dl & $\geq 4.0$ & $3.5-4.0$ & $3.0-3.5$ & $\leq 3.0$ \\
nPNA, mg/kg/day & $\geq 1.45$ & $1.18-1.45$ & $0.91-1.18$ & $\leq 0.91$ \\
Percent LBM, \% & $\geq 86.7$ & $73.2-86.7$ & $59.7-73.2$ & $\leq 59.7$ \\
\hline
\end{tabular}

equation: PNA $(\mathrm{g} / 24 \mathrm{~h})=10.76(0.69$ urea nitrogen appearance + 1.46). Urea nitrogen appearance was determined by measuring the urea in the patient's urine and dialysate. PNA was normalized to body weight (nPNA). LBM was estimated by creatinine kinetics. Total daily Cr production, measured as the sum of Cr excreted in urine and dialysate plus the estimated $\mathrm{Cr}$ lost via the gut, was used to calculate LBM by the following equation: $\mathrm{LBM}(\mathrm{kg})=$ $0.029 \times$ total Cr production $(\mathrm{mg} /$ day $)+7.38$. LBM was normalized by body weight (\%LBM).

\section{Definition of Protein Nutrition Index}

Serum albumin, nPNA and \%LBM were used to calculate PNI. For the serum albumin score, a score of 4 denoted albumin $\geq 4.0$ $\mathrm{g} / \mathrm{dl}$, a score of 3 denoted 3.5-4.0, a score of 2 denoted 3.0-3.5 and a score of $1 \leq 3.0$. The nPNA score and \%LBM were based on the mean value and 1 standard deviation (SD) of all patients. We defined the nPNA and \%LBM scores as follows: $4 \geq$ mean value +1 $\mathrm{SD}, 3$ = between mean $+1 \mathrm{SD}$ and mean, score $2=$ between mean and mean $-1 \mathrm{SD}$, score $1 \leq$ mean $-1 \mathrm{SD}$. For the nPNA score, a score of 4 denoted a nPNA $\geq 1.45 \mathrm{mg} / \mathrm{kg} /$ day, a score of 3 denoted 1.18-1.45, a score of 2 denoted $0.91-1.18$ and a score of 1 denoted $\leq 0.91$. For the $\%$ LBM score, a score of 4 denoted a $\%$ LBM $\geq 86.7 \%$, a score of 3 denoted $73.2-86.7 \%$, a score of 2 denoted $59.7-73.2 \%$ and a score of 1 denoted $\leq 59.7 \%$. The nutritional status of each patient was determined by their total score. Good nutrition was represented by a total score $>9$, high-average nutrition with a score of 8-9, low-average nutrition with a score of 6-7, and probable PEW with a score $<6$. The PNI are shown in table 1 .

\section{Comorbid Diseases}

Comorbid diseases were evaluated according to the categories used by Chung et al. [9]. Cardiovascular disease included a previous and present history of congestive heart failure, ischemic heart disease and cerebrovascular disease. Respiratory disease was defined as active tuberculosis, chronic lung disease or asthma attacks. Liver disease included persistently elevated serum glutamic-pyruvic transaminase and serum glutamic-oxaloacetic transaminase, or cirrhosis defined by abdominal echo and clinical symptoms. Systemic disease included diabetes mellitus and systemic lupus erythematosus.

Comorbidity was graded by the Davies index [10]. The comorbid score of each patient is determined by the number of comorbid diseases. Grade 0 (low risk) is a score of 0 , grade 1 (medium risk) is a score of $1-2$, and grade 2 (high risk) is a score $\geq 3$.

\section{Adequacy of $P D$ and the Peritoneal Equilibration Test}

Weekly total Kt/V urea (T-Kt/V) and weekly total creatinine clearance (T-Ccr) were calculated from dialysate and urine collected over a 24-hour period. The distribution volume of urea (V) 
Table 2. Baseline characteristics of different nutritional status in patients with $\mathrm{PD}(\mathrm{n}=552)$

\begin{tabular}{|c|c|c|c|c|c|}
\hline & $\begin{array}{l}\text { Good nutrition } \\
(\mathrm{n}=157)\end{array}$ & $\begin{array}{l}\text { High-average } \\
(\mathrm{n}=216)\end{array}$ & $\begin{array}{l}\text { Low-average } \\
(\mathrm{n}=115)\end{array}$ & $\begin{array}{l}\text { Probable PEW } \\
(\mathrm{n}=64)\end{array}$ & $\mathrm{p}$ \\
\hline Age, years & $42.6 \pm 11.7$ & $45.8 \pm 13.3^{*}$ & $51.8 \pm 14.1^{*, \dagger}$ & $63.2 \pm 13.7^{*, \dagger, \ddagger}$ & $<0.001$ \\
\hline Female sex, $\%$ & $100(63.7)$ & $127(58.8)$ & $72(62.6)$ & $41(64.1)$ & NS \\
\hline Comorbid index & $0.11 \pm 0.37$ & $0.30 \pm 0.51^{*}$ & $0.56 \pm 0.74^{*, \dagger}$ & $1.11 \pm 0.76^{*, \dagger, \ddagger}$ & $<0.001$ \\
\hline Serum BUN, mg/dl & $70.2 \pm 15.2$ & $62.2 \pm 16.2^{*}$ & $53.4 \pm 16.9^{*, \dagger}$ & $43.3 \pm 13.1^{*, \dagger, \ddagger}$ & $<0.001$ \\
\hline Serum Cr, mg/dl & $10.3 \pm 3.0$ & $9.9 \pm 2.8$ & $9.1 \pm 2.6^{*, \dagger}$ & $6.6 \pm 1.8^{*, \dagger, \ddagger}$ & $<0.001$ \\
\hline Total body water & $30.23 \pm 5.65$ & $31.06 \pm 5.48$ & $31.01 \pm 5.29$ & $29.33 \pm 4.37$ & NS \\
\hline 4-hour D/P Cr & $0.64 \pm 0.09$ & $0.67 \pm 0.10^{*}$ & $0.68 \pm 0.11^{*}$ & $0.72 \pm 0.12^{* \dagger, \ddagger}$ & $<0.001$ \\
\hline $\mathrm{K}-\mathrm{Kt} / \mathrm{V}$, urea/week & $0.73 \pm 0.51$ & $0.63 \pm 0.48^{*}$ & $0.48 \pm 0.39^{*, \dagger}$ & $0.45 \pm 0.35^{*, \dagger}$ & $<0.001$ \\
\hline $\mathrm{T}-\mathrm{Kt} / \mathrm{V}$, urea/week & $2.58 \pm 0.57$ & $2.37 \pm 0.52^{*}$ & $2.21 \pm 0.45^{*, \dagger}$ & $2.19 \pm 0.42^{* \dagger}$ & $<0.001$ \\
\hline $\mathrm{K}-\mathrm{Ccr}, 1 /$ week/1.73 m² & $31.7 \pm 22.1$ & $28.3 \pm 20.7$ & $21.9 \pm 17.2^{*, \dagger}$ & $22.1 \pm 17.3^{*, \dagger}$ & $<0.001$ \\
\hline T-Ccr, 1/week/1.73 m² & $78.3 \pm 21.0$ & $74.4 \pm 20.9$ & $68.1 \pm 17.2^{*, \dagger}$ & $70.3 \pm 20.2^{*}$ & $<0.001$ \\
\hline
\end{tabular}

was calculated with the Watson equation [11]. Weekly kidney $\mathrm{Kt} / \mathrm{V}$ urea (K-Kt/V) and weekly kidney Ccr (K-Ccr) represented residual renal function.

The peritoneal equilibration test was performed as described by Twardowski [28]. A standard 4-hour dwell was used, using a 2 -liter exchange with $2.27 \%$ glucose solution. The value for creatinine was corrected by subtracting the glucose concentration multiplied by a correction factor (0.0002) derived in our laboratory. Mean D4/P4 Cr was found to be $0.67 \pm 0.11$.

\section{Statistical Analysis}

The end point of the study was the patient status (dead or alive) at termination of the follow-up period. Patients were censored at transfer to hemodialysis or other centers, renal transplantation, or at the end of the observation period. Continuous variables were expressed as means $\pm \mathrm{SD}$. Analysis of variance (ANOVA) was used to compare differences between the four groups. Student's $t$ test was used to analyze the differences in variables in between survivors and nonsurvivors. The survival rates were determined by the Kaplan-Meier method. A log-rank test was used to compare the different survival curves. The Cox proportional hazards model was utilized to analyze factors predicting mortality and the risk ratios for mortality. The difference was considered significant with $\mathrm{p}<0.05$. All statistical calculations were performed using SPSS 12.0 for Windows.

\section{Results}

Clinical Characteristics of Patients at Initiation of PD

Among 552 PD patients, 340 were women and the mean age of all patients was $48.2 \pm 14.5$ years (range 18-87). The major diagnosis of renal failure was glomerulonephritis (24\%, 133 patients) and diabetes mellitus ne- phropathy (18\%, 98 patients). 171 patients had 1 or more comorbid diseases for a total of 199 events: 98 patients with diabetes mellitus, 62 with cardiovascular disease, 3 with respiratory disease, 18 with liver disease and 18 with systemic lupus erythematosus. Based on the Davies index, 381 patients were classified as low-risk, 170 patients as medium-risk group and 1 patient as high-risk. 478 patients $(86.6 \%)$ received continuous ambulatory peritoneal dialysis (CAPD) as their dialysis mode.

\section{Distribution of PNI in Patients at the Start of PD}

At the start of PD, among 552 patients, 157 patients were classified as having good nutrition, 216 patients as high-average nutrition, 115 patients as low-average nutrition and 64 patients as probable PEW. Age, sex, comorbid index, D/P Cr (dialysate-to-plasma Cr ratio), Kt/V and $\mathrm{Ccr}\left(1 /\right.$ week $\left./ 1.73 \mathrm{~m}^{2}\right)$ were compared between groups according to initial nutritional status using ANOVA. There were significant differences between groups in the case of every parameter except distribution of sex (table 2). $\mathrm{Pa}-$ tients with probable $\mathrm{PEW} /$ low-average nutrition were older and had lower serum $\mathrm{Cr}$ and BUN concentrations, T-Kt/V, K-Kt/V, T-Ccr, and K-Ccr (1/week/1.73 m²), but higher D4/P4 Cr, compared to patients with high-average or good nutrition.

\section{Determinants of PNI in Patients at the Start of PD}

By simple-linear regression analysis, age, comorbid index, serum Cr, D4/P4 Cr, T-Kt/V and K-Kt/V were associated with PNI in patients at the start of PD. After adjust- 
Table 3. Determinants of PNI at the start of PD $(n=552)$

\begin{tabular}{lrrrrr}
\hline & \multicolumn{2}{c}{ Simple linear regression } & & \multicolumn{2}{c}{ Stepwise multiple linear regression } \\
\cline { 2 - 3 } & $\mathrm{r}$ & $\mathrm{p}$ & & $\beta$-coefficient $\pm \mathrm{SE}$ & $\mathrm{p}$ \\
\hline Age & -0.431 & $<0.0001$ & & $-0.159 \pm 0.005$ & $<0.0001$ \\
Comorbid index & -0.485 & $<0.0001$ & & $-0.209 \pm 0.104$ & $<0.0001$ \\
Serum Cr & 0.356 & $<0.0001$ & & $0.526 \pm 0.028$ & $<0.0001$ \\
4-hour D/P Cr & -0.210 & $<0.0001$ & & $-0.076 \pm 0.582$ & 0.014 \\
T-Kt/V & -0.306 & $<0.0001$ & & $0.203 \pm 0.195$ & $<0.0001$ \\
K-Kt/V & 0.280 & $<0.0001$ & & $0.368 \pm 0.182$ & $<0.0001$ \\
\hline
\end{tabular}

Table 4. Clinical factors, PNI and adequacy of peritoneal dialysis in survivors and nonsurvivors $(\mathrm{n}=552)$

\begin{tabular}{lccc}
\hline & $\begin{array}{l}\text { Survivors } \\
(\mathrm{n}=444)\end{array}$ & $\begin{array}{l}\text { Nonsurvivors } \\
(\mathrm{n}=108)\end{array}$ & $\mathrm{p}$ \\
\hline Age, years & $44.9 \pm 13.0$ & $61.6 \pm 12.4$ & $<0.001$ \\
Comorbid index & $0.23 \pm 0.48$ & $1.07 \pm 0.77$ & $<0.001$ \\
Serum Cr, mg/dl & $9.76 \pm 2.84$ & $8.28 \pm 3.07$ & $<0.001$ \\
PNI & $8.56 \pm 1.74$ & $6.64 \pm 2.21$ & $<0.001$ \\
4-hour D/P Cr & $0.67 \pm 0.10$ & $0.67 \pm 0.12$ & $\mathrm{NS}$ \\
K-Kt/V, urea/week & $0.64 \pm 0.48$ & $0.47 \pm 0.36$ & $<0.001$ \\
T-Kt/V, urea/week & $2.41 \pm 0.54$ & $2.24 \pm 0.49$ & 0.003 \\
K-Ccr, 1/week/1.73 m m $^{2}$ & $28.33 \pm 20.74$ & $22.65 \pm 18.33$ & 0.009 \\
T-Ccr, 1/week/1.73 m & $74.80 \pm 20.26$ & $69.38 \pm 19.61$ & 0.012 \\
Total body water & $30.79 \pm 5.44$ & $29.87 \pm 5.13$ & $\mathrm{NS}$ \\
\hline
\end{tabular}

ing for the above significant variables via multiple linear regression analysis along with backward stepwise elimination, age, comorbid index, serum $\mathrm{Cr}, \mathrm{D} 4 / \mathrm{P} 4 \mathrm{Cr}$, T-Kt/V and $\mathrm{K}-\mathrm{Kt} / \mathrm{V}$ were all determinants of PNI in PD patients (table 3).

\section{Clinical Outcomes (Patient Survival)}

The end point of the study was the patient status (dead or alive) at termination of the follow-up period (mean duration of $\mathrm{PD} 45.3 \pm 30.2$ months, range 3.4-147), at which point 108 patients had died, 46 patients had received renal transplantation, 126 patients had been transferred to hemodialysis and 32 patients had been transferred to other units. Among the patients who died, 60 (55.6\%) died of infection, $40(40 / 108 ; 37 \%)$ died of cardiovascular disease and 8 died of other causes. Patients who died during the follow-up period were older and had a higher comorbid index and lower PNI compared to survivors (table 4).

With the Kaplan-Meier analysis, the patient survival rate was significantly lower in patients with probable PEW and lower-average nutrition than those with high- average and good nutrition ( $\mathrm{p}=0.001$; fig. 1 ). The 5 -year patient survival rate was about $26,66,87$ and $92 \%$ for subgroups with probable PEW, low-average nutrition, highaverage nutrition and good nutrition, respectively.

\section{Predictors of Patient Survival and Risk Ratio for Mortality}

Predictors of mortality and RR for mortality are shown in table 5. By Cox proportional hazards univariate analysis, age, comorbid index, PNI, T-Kt/V and $\mathrm{K}-\mathrm{Kt} / \mathrm{V}$, and T-Ccr and K-Ccr $\left(1 /\right.$ week/1.73 $\left.\mathrm{m}^{2}\right)$ were predictors of mortality. However, by multivariate analysis, only age $(\mathrm{RR}=1.07,95 \%$ CI: $1.05-1.08, \mathrm{p}<0.001)$, comorbidity index $(\mathrm{RR}=2.60,95 \% \mathrm{CI}: 2.0-3.38, \mathrm{p}<0.001)$ and PNI $(\mathrm{RR}=0.84,95 \% \mathrm{CI}: 0.76-0.93, \mathrm{p}=0.001)$ were found to be independent predictors of mortality (table 5).

\section{Discussion}

The present study revealed that PNI at the start of dialysis can predict long-term survival in $\mathrm{PD}$ patients, even after adjusting for age, comorbidity and adequacy of dialysis. This finding emphasizes that nutritional status at the start of PD has a great impact on the long-term survival of patients, and PEW increases all-cause mortality.

Besides serum albumin, the scoring system of PNI was based on the mean \pm SD of nPNA (mg/kg/day) and $\%$ LBM of our population (552 patients). For the nPNA score in our study, a score of 2 denoted 0.91-1.18 and a score of $1 \leq 0.91$. According to a previous guide, nPNA $<1.2$ suggested malnutrition and $<0.8$ meant severe malnutrition [12]. The value of the nPNA score is very close to the general PD population. When comparing to nPNA as the standard (nPNA $<0.9$ as malnutrition), the sensitivity, specificity, positive and negative predictive value of PNI was $0.4,0.978,0.901$ and 0.783 , respectively. 
Fig. 1. Kaplan-Meier survival curve of patients with different PNI values. The patient survival rate was significantly lower in patients with probable PEW and loweraverage nutrition than high-average and good nutrition $(\mathrm{p}<0.01)$.

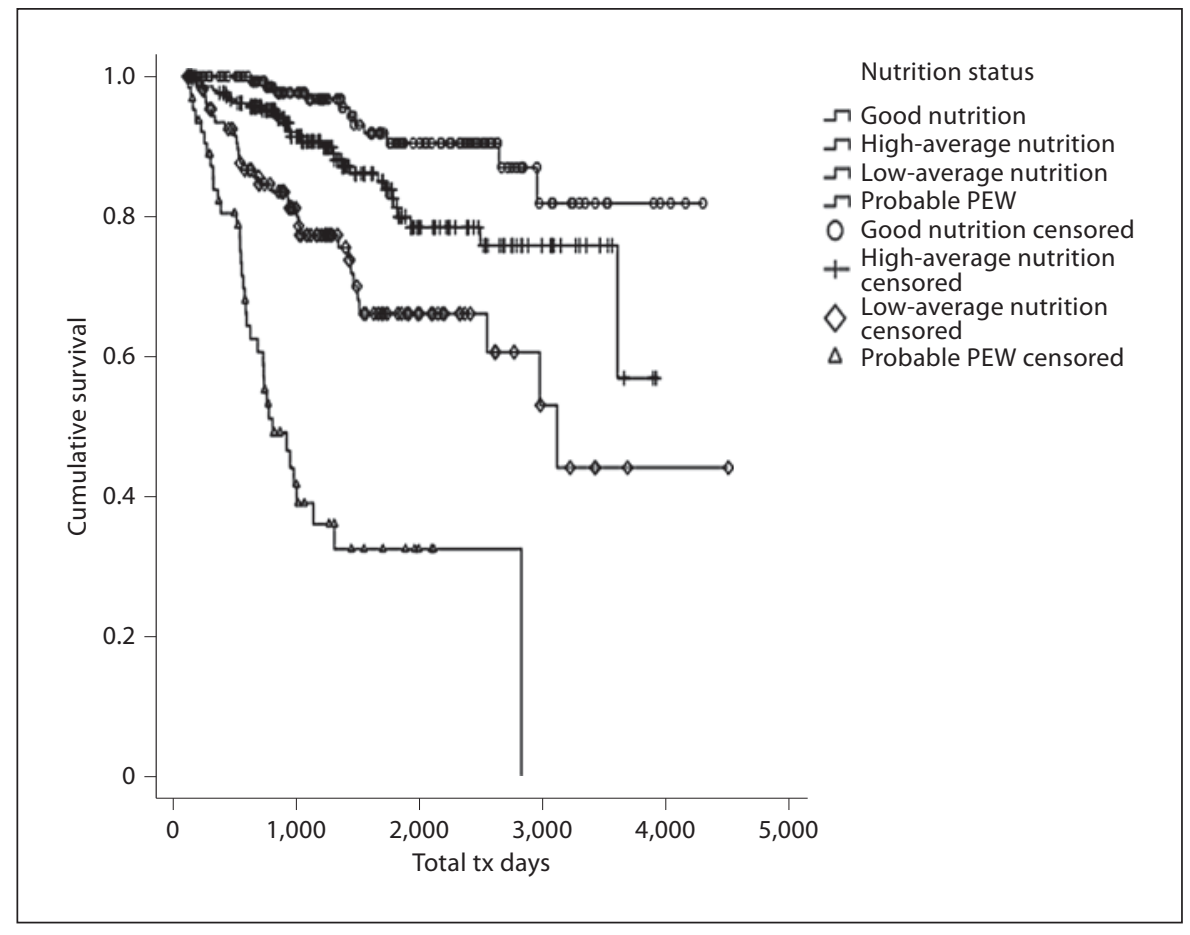

Table 5. Cox regression analysis of the overall risk of all-cause mortality, according to baseline prognostic factors in PD patients $(\mathrm{n}=552)$

\begin{tabular}{llllr}
\hline Variable & $\begin{array}{l}\text { Univariate hazard analysis } \\
\text { RR }(95 \% \mathrm{CI})\end{array}$ & $\mathrm{p}$ & $\begin{array}{l}\text { Multivariate hazard analysis } \\
\text { RR }(95 \% \mathrm{CI})\end{array}$ & $\mathrm{p}$ \\
\hline Age & $1.09(1.08-1.11)$ & $<0.001$ & $1.07(1.05-1.08)$ & $<0.001$ \\
Comorbid index & $4.44(3.54-5.57)$ & $<0.001$ & $2.60(2.0-3.38)$ & $<0.001$ \\
PNI & $0.62(0.57-0.68)$ & $<0.001$ & $0.84(0.76-0.93)$ & \\
Serum Cr & $0.82(0.75-0.89)$ & $<0.001$ & \\
4-h D/P Cr & $1.84(0.31-10.86)$ & 0.503 & \\
K-Kt/V & $0.42(0.26-0.67)$ & $<0.001$ & \\
T-Kt/V & $0.47(0.31-0.70)$ & $<0.001$ & \\
K-Ccr & $0.99(0.98-0.99)$ & 0.008 & \\
T-Ccr & $0.99(0.98-0.99)$ & 0.008 & \\
\hline
\end{tabular}

Several studies with \%LBM evaluation in their reports were reviewed. In the study by Chung et al. [13], the \%LBM of patient with medium risk by comorbidity grade was $59.4 \pm 14.3$. In another study by Szeto et al. [14], \%LBM in the interventional group was $66.0 \pm 10.7$. In our \%LBM score, a score of 2 denoted 59.7-73.2 and a score of 1 denoted $\leq 59.7$. The value of the \%LBM score is also very close to other groups of PD patients. Therefore, the values of score 2 and 1 in nPNA and \%LBM are similar to other studies about PD. In diagnosis of PEW, the score can be applicable to the worldwide PD population.

By comparing patients with good nutrition, high-average nutrition, low-average nutrition and probable PEW, it was found that poor nutritional status was associated with older age, more comorbidity, lower serum $\mathrm{Cr}$ and BUN levels, higher D4/P4 Cr, and a lower value of PD adequacy measures. Factors determining baseline PNI were further investigated. Multiple linear regression analysis demonstrated that age, comorbidity, serum Cr, D4/P4 Cr, 
$\mathrm{T}-\mathrm{Kt} / \mathrm{V}$ and $\mathrm{K}-\mathrm{Kt} / \mathrm{V}$ were factors independently associated with PNI at the start of PD.

Age is an important factor influencing the nutritional status. Aging causes a general decline in the function of the gastrointestinal tract, and age-associated malabsorption of nutrients may be one of several factors that contribute to the malnutrition that occurs with aging [15]. Comorbidities surely have negative effects on the nutritional status. In PD patients, diabetes and cardiovascular disease are the most common comorbidities. Wang et al. [16] proved that background cardiac disease was associated with poor nutritional status in CAPD patients. Serum $\mathrm{Cr}$ levels are a measure of the muscle mass status and reflect the nutritional condition of dialysis patients [17]. A higher $\mathrm{Cr}$ level indicates increased muscle mass and better nutrition. Kang et al. [18] reported that peritoneal membrane transport status (D4/P4 Cr) correlated with the overall nutritional status of $\mathrm{PD}$ patients, and they suggested that a high permeability state is a risk factor for malnutrition in CAPD patients. Szeto et al. [19] also found that baseline D4/P4 Cr correlated negatively with serum albumin, PNA and \%LBM, although after 2 years, peritoneal transport status was not found to be associated with longitudinal change in nutritional parameters. Hence, our results were in agreement with previous studies: $\mathrm{D} 4 / \mathrm{P} 4 \mathrm{Cr}$ at the start of $\mathrm{PD}$ is an important factor associated with baseline nutritional status.

In previous studies, weekly $\mathrm{T}-\mathrm{Kt} / \mathrm{V}$ and residual renal function $(\mathrm{K}-\mathrm{Kt} / \mathrm{V})$ have been found to be related to nutritional status. Nolph et al. [20] reported that weekly Kt/V urea correlated with nPNA and serum albumin, and they concluded that greater small solute clearances were associated with better nutritional status. Low residual renal function at the start of $\mathrm{PD}$, evaluated by initial renal Kt/V urea, was associated with lower serum albumin, nPNA and \%LBM [21].

At the end of the observation period, a total of $108 \mathrm{pa}-$ tients had died, and the overall mortality was $23.2 \%$. Cox multivariate analysis revealed that age, comorbid index and PNI were significant risk factors for all-cause mortality in PD patients. It was found that a 1-year increase in age resulted in a $7 \%$ increased risk of mortality. A further increase in the comorbid index by a score of 1 resulted in a $160 \%$ increased risk of mortality, and an increase in PNI by a score of 1 led to a $16 \%$ decreased risk of mortality. This finding further emphasized that baseline nutritional status at the start of PD can predict long-term mortality during PD, independent of age and comorbidity.

Nutritional status is associated with clinical outcome in dialysis patients. In the CANUSA study, better nutri- tion was associated with better patient survival and fewer hospitalization days, while serum albumin concentration and \%LBM were associated with mortality [2]. A difference of $1 \mathrm{~g} / \mathrm{dl}$ of serum albumin was associated with a 6\% change in the RR of death, and a $1 \%$ difference in \%LBM was associated with $3 \%$ change in the RR of death [2].

Evidence linking survival of PD patients with nutritional status at initiation of PD has been noted in several studies. Hakim and Lazarus [5] found that malnutrition at the start of dialysis is a strong predictor of subsequent increase in RR of death. Chung et al. [22] also suggested that initial nutritional status, measured by fat-free edema-free body mass and SGA, is an independent predictor of death [22]. PEW is often seen in patients at the start of PD. Pollock et al. [23] found that a lower dietary protein intake correlated with level of renal dysfunction, and this change resulted in PEW at the start of dialysis.

PEW is also associated with inflammation and atherosclerosis in uremic patients $[3,4,24,25]$. Therefore, before dialysis is initiated, chronic inflammation and progressive atherosclerosis have already happened in patients with PEW. This change may not be reversed by dialysis. Cardiovascular disease is the most important cause of mortality in dialysis patients. Thus, initial nutritional status may be linked to patients' survival rates during dialysis.

Dialysis adequacy at start of PD was not an independent risk factor of mortality in this study. According to the ADEMEX study, dialysis adequacy as determined by small solute clearance may not be as critical as previously thought for the clinical outcome of PD patients [26]. Li and Szeto [27] suggested a target Kt/V total of 1.9 in Asian CAPD populations. In patients with probable PEW in our study, T-Kt/V was about 2.2. Therefore, baseline PD adequacy was not a risk factor for mortality in our groups.

There are several limitations to our analysis. First, we did not use SGA, anthropometric or bioimpedance indices analysis to evaluate nutritional status in this study. Because of technical difficulty in follow-up and variations among observers, SGA was not routinely assessed in our PD unit. In addition, the measurement of SGA did not diagnose malnutrition in any patient in whom malnutrition had not already been potentially identified by measurement of serum albumin or other nutritional markers [8]. However, it is necessary to demonstrate a positive correlation between PNI and SGA by anthropometric analysis in the future. Additionally, the reliability of the PNI needs to be confirmed by more studies in the future. Second, inflammatory status was not evaluated in this study. Inflammation contributes to PEW in dialysis 
patients. We need further confirmation regarding the relationship between inflammatory status and PNI in subsequent studies.

In conclusion, PEW at the start of PD predicts longterm mortality in patients. PNI is an objective method for evaluating nutritional status of dialysis patients. PNI at the start of PD is associated with long-term all-cause mortality, even after adjusting for age and comorbidity. An increase in PNI by a score of 1 leads to a 16\% decreased risk of mortality. Predialysis evaluation of this scoring sys- tem is recommended for further research in order to improve outcomes in PD patients. Future studies should further compare PNI with other nutritional evaluating systems and investigate its relationship with inflammation.

\section{Acknowledgment}

We appreciate the help provided by the PD staff of Lin-Kou Chang Gung Memorial Hospital.

\section{References}

1 Fouque D, Kalantar-Zadeh K, Kopple J, Cano N, Chauveau P, Cuppari L, Franch H, Guarnieri G, Ikizler TA, Kaysen G, Lindholm B, Massy Z, Mitch W, Pineda E, Stenvinkel P, Treviño-Becerra A, Wanner C: A proposed nomenclature and diagnostic criteria for protein-energy wasting in acute and chronic kidney disease. Kidney Int 2008;73:391-398.

$\checkmark 2$ Adequacy of dialysis and nutrition in continuous peritoneal dialysis: Association with clinical outcomes. Canada-USA (CANUSA) peritoneal dialysis study group. J Am Soc Nephrol 1996;7:198-207.

-3 de Mutsert R, Grootendorst DC, Axelsson J, Boeschoten EW, Krediet RT, Dekker FW; NECOSAD Study Group: Excess mortality due to interaction between protein-energy wasting, inflammation and cardiovascular disease in chronic dialysis patients. Nephrol Dial Transplant 2008;23:2957-2964.

4 Rambod M, Bross R, Zitterkoph J, Benner D, Pithia J, Colman S, Kovesdy CP, Kopple JD, Kalantar-Zadeh K: Association of malnutrition-inflammation score with quality of life and mortality in hemodialysis patients: a 5-year prospective cohort study. Am J Kidney Dis 2009;53:298-309.

$\checkmark 5$ Hakim RM, Lazarus JM: Initiation of dialysis. J Am Soc Nephrol 1995;6:1319-1328.

-6 de Mutsert R, Grootendorst DC, Boeschoten EW, Brandts H, van Manen JG, Krediet RT, Dekker FW; Netherlands Cooperative Study on the Adequacy of Dialysis-2 Study Group: Subjective global assessment of nutritional status is strongly associated with mortality in chronic dialysis patients. Am J Clin Nutr 2009;89:787-793.

$>7$ Szeto CC, Kong J, Wu AK, Wong TY, Wang AY, Li PK: The role of lean body mass as a nutritional index in Chinese peritoneal dialysis patients - comparison of creatinine kinetics method and anthropometric method. Perit Dial Int 2000;20:708-714.

-8 Gurreebun F, Hartley GH, Brown AL, Ward MC, Goodship TH: Nutritional screening in patients on hemodialysis: is subjective global assessment an appropriate tool? J Ren Nutr 2007; 17:114-117.
-9 Chung SH, Lindholm B, Lee HB: Is malnutrition an independent predictor of mortality in peritoneal dialysis patients? Nephrol Dial Transplant 2003;18:2134-2140.

10 Davies SJ, Phillips L, Naish PF, Russell GI: Quantifying comorbidity in peritoneal dialysis patients and its relationship to other predictors of survival. Nephrol Dial Transplant 2002;17:1085-1092.

11 Watson PE, Watson ID, Batt RD: Total body water volumes for adult males and females estimated from simple anthropometric measurements. Am J Clin Nutr 1980;33:27-39.

12 Abbas K: Using normalized protein nitrogen appearance (nPNA) in assessing nutrition. Nephrol Nurs J 2006;33:677-678.

13 Chung SH, Lindholm B, Lee HB: Is malnutrition an independent predictor of mortality in peritoneal dialysis patients? Nephrol Dial Transplant 2003;18:2134-2140.

14 Szeto CC, Wong TY, Chow KM, Leung CB, Wang AY, Lui SF, Li PK: The impact of increasing the daytime dialysis exchange frequency on peritoneal dialysis adequacy and nutritional status of Chinese anuric patients. Perit Dial Int 2002;22:197-203.

15 Drozdowski L, Thomson AB: Aging and the intestine. World J Gastroenterol 2006;12: 7578-7584.

16 Wang AY, Sea MM, Ng K, Kwan M, Lui SF, Woo J: Nutrient intake during peritoneal dialysis at the Prince of Wales Hospital in Hong Kong. Am J Kidney Dis 2007;49:682-692.

17 Thunberg BJ, Swamy AP, Cestero RV: Cross sectional and longitudinal nutritional measurements in maintenance hemodialysis patients. Am J Clin Nutr 1981;34:2005-2012.

18 Kang DH, Yoon KI, Choi KB, Lee R, Lee HY, Han DS, Cho EY, Lee JH: Relationship of peritoneal membrane transport characteristics to the nutritional status in CAPD patients. Nephrol Dial Transplant 1999; 14: 1715-1722.

19 Szeto CC, Law MC, Wong TY, Leung CB, Li PK: Peritoneal transport status correlates with morbidity but not longitudinal change of nutritional status of continuous ambulatory peritoneal dialysis patients: a 2-year prospective study. Am J Kidney Dis 2001;37: 329-336.
20 Nolph KD, Moore HL, Prowant B, Meyer M, Twardowski ZJ, Khanna R, Ponferrada L, Keshaviah P: Cross sectional assessment of weekly urea and creatinine clearances and indices of nutrition in continuous ambulatory peritoneal dialysis patients. Perit Dial Int 1993;13:178-183.

21 Misra M, Nolph KD, Khanna R, Prowant BF, Moore HL: Retrospective evaluation of renal $\mathrm{kt} / \mathrm{V}$ (urea) at the initiation of long-term peritoneal dialysis at the University of Missouri: relationships to longitudinal nutritional status on peritoneal dialysis. ASAIO J 2003;49: 91-102.

22 Chung SH, Lindholm B, Lee HB: Influence of initial nutritional status on continuous ambulatory peritoneal dialysis patient survival. Perit Dial Int 2000;20:19-26.

23 Pollock CA, Ibels LS, Zhu FY, Warnant M, Caterson RJ, Waugh DA, Mahony JF: Protein intake in renal disease. J Am Soc Nephrol 1997;8:777-783.

24 Pupim LB, Caglar K, Hakim RM, Shyr Y, Ikizler TA: Uremic malnutrition is a predictor of death independent of inflammatory status. Kidney Int 2004;66:2054-2060.

25 Pecoits-Filho R, Nordfors L, Lindholm B, Hoff CM, Schalling M, Stenvinkel P: Genetic approaches in the clinical investigation of complex disorders: malnutrition, inflammation, and atherosclerosis (MIA) as a prototype. Kidney Int Suppl 2003:S162-S167.

$>26$ Paniagua R, Amato D, Vonesh E, CorreaRotter R, Ramos A, Moran J, Mujais S: Effects of increased peritoneal clearances on mortality rates in peritoneal dialysis: ADEMEX, a prospective, randomized, controlled trial. J Am Soc Nephrol 2002; 13:1307-1320.

27 Li PK, Szeto CC: Adequacy targets of peritoneal dialysis in the Asian population. Perit Dial Int 2001;21(Suppl 3):S378-S383.

28 Twardowski ZJ: Clinical value of standardized equilibration tests in CAPD patients. Blood Purif 1989;7:95-108. 